\title{
2.I Long-term safety and efficacy of tocilizumab in children with systemic juvenile idiopathic arthritis (JIA)
}

S Yokota*1, T Imagawa ${ }^{1}$, T Miyamae ${ }^{1}$, K Kasai ${ }^{1}$, M Mori ${ }^{1}$, N Nishimoto ${ }^{2}$ and T Kishimoto ${ }^{2}$

Address: ${ }^{1}$ Yokohama City University, Yokohama, Kanagawa, Japan and ${ }^{2}$ Osaka University, Suita, Osaka, Japan

* Corresponding author

from 15 th Paediatric Rheumatology European Society (PreS) Congress

London, UK. 14-I7 September 2008

Published: 15 September 2008

Pediatric Rheumatology 2008, 6(SuppI I):SI doi:I0.II86/I546-0096-6-SI-SI

This abstract is available from: http://www.ped-rheum.com/content/6/SI/SI

(C) 2008 Yokota et al; licensee BioMed Central Ltd.

\section{Objectives}

To evaluate long-term safety and efficacy of tocilizumab in treatment of children with systemic JIA.

\section{Methods}

Patients with systemic JIA fulfilled WHO/ILAR criteria were enrolled in long-term extension study immediately after completion of phase II and III trials of tocilizumab. Tocilizumab was intravenously administered at a dose of $8 \mathrm{mg} / \mathrm{kg}$ every 2 weeks. Efficacy was assessed every 6 weeks using ACR Pedi 30 Criteria for Improvement.

\section{Results}

Sixty seven patients (29 boys and 38 girls) were included in this analysis. Median age was 8 years and median disease duration was 3.8 years. At the time of analysis, 9 patients had discontinued tocilizumab treatment, 4 due to AEs, 4 due to development of anti-tocilizumab antibodies and 1 due to lack of efficacy. Median duration of tocilizumab treatment was 146 weeks. The most frequent AEs were upper respiratory tract infections and gastroenteritis. The incidence rate of serious infections was 10.7 per 100 patient-years. No deaths, malignancies, or autoimmune diseases were observed. ACR Pedi 30, 50 and 70 Improvement Criteria were achieved in 100\%, $98 \%$ and $93 \%$ at Week 96. All patients were treated with oral corticosteroids at the registration and $72 \%$ were able to reduce corticosteroid dose by more than $50 \%$ at Week 96. Fourteen patients became steroid-free during the study. 4 patients were in remission without tocilizumab itself and any other medications.

\section{Conclusion}

The long-term extension study demonstrated sustained clinical improvement and a favourable risk-benefit profile for tocilizumab treatment in children with systemic JIA. Even medication-off status will be expected. 